Revista Iberoamericana, Vol. LXXVII, Núm. 235, Abril-Junio 2011, 461-471

\title{
NANCY MOREJÓN Y SU POÉTICA DEL CUERPO EN LA CLARIDAD Y LA PENUMBRA
}

\author{
POR \\ Flora GonZÁlez Mandri \\ Emerson College
}

En su introducción a la antología crítica Recovering the BlackFemale Body, Michael Bennett y Vanessa D. Dickerson explican:

This collection attempts to carefully intervene in the recently emergent areas of the literary and theoretical study of the black female body without reinscribing the dominant discourse's negative construction of African American women or turning the black female body into a spectacle toward some other end. (5)

[Esta colección intenta intervenir meticulosamente en las áreas emergentes de los estudios literarios y teóricos del cuerpo de la mujer negra sin reinscribir en él el discurso dominante con su construcción negativa de la mujer afroamericana o sin convertir el cuerpo de la mujer negra en un espectáculo con otros fines. $]^{1}$

Entonces, ¿cómo es que la escritora de la diáspora africana interviene para alumbrar el cuerpo femenino sin convertirlo en un espectáculo para ser consumido por la mirada colonizante? La poeta cubana Nancy Morejón realiza una reintegración del cuerpo de la mujer negra personificándolo dentro del contexto histórico de la trata a través del Atlántico. Situándolo dentro del contexto martiano que declara "Dos patrias tengo yo: Cuba y la noche”, Morejón crea el cuerpo de la mujer negra en la claridad del mar Caribe y en la penumbra de la noche: "Mi cuerpo en la penumbra/ Mi cuerpo en claridad” (Looking 156).

En estas páginas hemos de concentrarnos en una serie de poemas donde el cuerpo de la mujer negra, históricamente relegado a la penumbra de la servitud, ha de ser celebrado mediante metáforas vinculadas a las fuerzas dinámicas de la naturaleza, fuerzas que lo localizan a la luz de la cultura afrocubana. En las primeras páginas de su gran compendio de testimonios sobre la cultura afrocubana, El monte, Lydia Cabrera sienta

1 Todas las traducciones al español son mías. 
varios conceptos fundamentales: "Persiste en el negro cubano, con tenacidad asombrosa, la creencia en la espiritualidad del monte" (13). Cabrera declara que esta espiritualidad del monte existe "todavía hoy, igual que en los días de la trata” (13). Además añade, "Monte equivale a Tierra en el concepto de Madre universal, fuente de vida. 'Tierra y monte es lo mismo’” (13). Para el propósito del análisis que sigue, hemos de retener la equivalencia entre Monte, lugar sagrado, y Tierra, "Madre universal, fuente de vida". Dentro del contexto que nos presenta Cabrera, también existe la presencia de lo espiritual y lo sagrado en el presente de un espacio (Tierra, Monte) que contiene en sí "los días de la trata”. Proponemos entonces que en el espacio de la poesía de Morejón, Tierra o naturaleza puede servirnos de metáfora para el hacer poético ("fuente de vida"), capaz de hacer coincidir el hoy de la enunciación y la herencia histórica de una cultura ("los días de la trata").

En "El tambor”, Nancy Morejón nos ofrece el cuerpo femenino “en la luz/ vuestra, libre, en el arco", presentando a la luz del lector una serie de símiles y metáforas que relacionan el cuerpo de la mujer con las islas del Caribe ("como islas"), y toda la cosmología circundante ("el coral”, "mis aguas”, “mi bruma”, y en "los azules de mi tierra") (Looking 156). La primera parte de "El tambor" establece los contornos del cuerpo y sus poderes: "Mi cuerpo convoca la llama./ Mi cuerpo convoca los humos" (156). El cuerpo físico/poético empieza convocando "la llama” y "los humos" en el sentido de dar voces que alumbran y oscurecen, así haciendo eco al título "El tambor", que dentro del contexto Bantú afrocubano nombra creando con su sonido retumbante. En efecto, la voz hablante nombra el cuerpo femenino, y por virtud de la relación entre el acto de convocar y el tambor, Nancy Morejón describe un vínculo entre el quehacer poético de dar voz y el resonar del tambor africano (metáfora del cuerpo femenino y símbolo de la cultura afrocubana). En este sentido, cuerpo femenino y cultura afrocubana coexisten en el lugar de la metáfora.

En su estudio sobre "Sensemayá” de Nicolás Guillén, Eugenio Matibag define el término Bantú nommo: "Connota la identificación marcada entre la persona o cosa y el nombre de la persona o cosa” (158-59). A partir del título, el poema traza la interrelación entre la palabra tambor y el cuerpo propio de la mujer, que al resonar como un tambor crea el archipiélago cubano ("mi cuerpo como islas"). Si en el poema "Mirar adentro" Morejón utiliza la metáfora eurocéntrica del ruiseñor para significar la voz del poeta, "porque aquel ruiseñor/ siempre canta mi pena” (Looking 160), en “El tambor” la poeta nombra la herencia africana desplazando la voz cantante para hacer relucir el cuerpo resonante: "Mi cuerpo ingrávido en la luz/ vuestra, libre, en el arco" (Looking 156). Pero el cuerpo que antes fue tambor es, en los últimos versos, isla en su arco libre de todo el peso de su historia esclavizante ("Mi cuerpo en el desastre" -"los días de la trata" mencionados en El monte-). Aún en su estado ingrávido, libre, "Mi cuerpo" aparece en toda su plenitud geográfica en tanto isla que traza un arco en el mar Caribe.

\footnotetext{
Revista Iberoamericana, Vol. LXXVII, Núm. 235, Abril-Junio 2011, $461-471$
ISSN 2154-4794 (Electrónico)
} 
En el poema "Pelo" el cuerpo de la mujer adquiere rasgos mucho más concretos, personales, con el pelo blanco significando el pasaje del tiempo, pero manteniendo una vitalidad engendradora, propia del concepto lucumí del Monte, que significa lugar sagrado, fuente de vida:

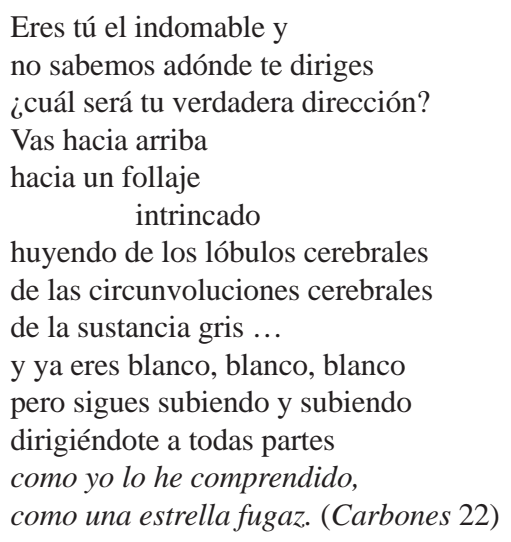

Aunque forma sólo parte del cuerpo de la mujer, el pelo lo cubre y exige ser nombrado en la poesía: "siempre has/ querido, entrar en mis/ versos que no son mucho más/ suaves que tú”. Con estos versos la voz de la hablante rechaza la naturaleza de su pelo a partir de los prejuicios raciales que le enseñaron a odiarlo: "Me cubres y eres mío/ y porque me enseñaron/ a odiarte/ eres más mío" (22). El pelo, indomable, crece hacia arriba, "hacia un follaje/ intrincado/ huyendo de los lóbulos cerebrales". El pelo rechaza todo prejuicio racionalizado por seres esclavizantes, e insiste en su energía vegetal, en su color blanco, para reverberar en las alturas, “como una estrella fugaz”. Nancy Morejón reconstruye un significado alternativo para el cuerpo de la mujer negra que borra todas las connotaciones negativas asociadadas al cuerpo negro desde el tiempo de la esclavitud, ubicando así la escena del pelo "indomable” como acto de rebelión ante los prejuicios de la sociedad europeizante.

En su ensayo sobre la representación de la mujer negra a través de su pelo, en relación a la novela Dessa Rose de Sherley Anne Williams, Noliwe Rooks habla de la costumbre de mujeres de varias generaciones que se encuentran para arreglarse el pelo y para conversar y hacer cuentos de familia:

As a result, this scene in Dessa Rose highlights one of the truly important aspects of hair as it relates to African American culture: the act of styling hair as both a social and learning occasion. (286)

Revista Iberoamericana, Vol. LXXVII, Núm. 235, Abril-Junio 2011, $461-471$
ISSN 2154-4794 (Electrónico) 
[Como resultado, esta escena en Dessa Rose ilumina uno de los aspectos más importantes del pelo en relación a la cultura afroamericana: el acto de arreglarse el pelo como ocasión social y pedagógica.]

Aunque Morejón no reproduce esa escena comunitaria en "Pelo", sí lo hace si lo consideramos en conversación con el conocido poema a su abuela paterna, "Presente Brígida Noyola” (Looking 212). Aquí, como en "Pelo”, se establece un vínculo entre el cuerpo de la mujer negra y los poderes dinámicos de la naturaleza. Noténse el primer y último versos: "tú eres grano y volcán” y “como la tierra creces tú”. El cuerpo de la mujer negra está representado al principio del primer verso mediante la metáfora del grano, forma vegetativa minúscula con capacidades de romper el terreno para dar a luz una planta. En la segunda parte del mismo verso, el poder germinativo de la semilla adquiere fuerzas telúricas cuando se le iguala al volcán, así asociando al cuerpo femenino la capacidad de ocupar un espacio trascendente, desde el centro de la tierra hasta más allá de las nubes. El pelo de la "abuela materna” ocupa la segunda estrofa del poema: "tu pelo largo negro/ nace desde la frente opaca/ y llega hasta la boca/ menuda ..." (212). Los verbos "nacer” y "llegar" acentúan el mismo movimiento establecido en el primer verso, con la intención de hacer hincapié en la capacidad del pelo de crecer, en todas sus connotaciones literales y metafóricas. Cabe mencionar que el poema empieza con un "tú" en letra minúscula para acentuar el poder germinativo concentrado en un cuerpo familiar, un cuerpo con el que la voz hablante del poema puede establecer un vínculo genealógico.

En estos poemas existe un desdoblamiento de "el pelo" y “mi cuerpo" a través del uso de la personificación, con el cuerpo capaz de convocar la llama y los humos y el pelo naciendo, llegando, y queriendo incorporarse a los versos. Este distanciamiento retórico sirve para inscribir el cuerpo de la mujer negra dentro de la cosmología afrocubana (el cuerpo/tambor; el pelo/follaje/Monte). Esta visión del cuerpo de la mujer negra también se sitúa dentro del marco histórico que en "El tambor" incluye la subyugación del cuerpo femenino al "desastre" del rapto, el trabajo forzado y la violación sexual. Ese cuerpo también sufrió el colonialismo de las "catedrales” que estableció la equivalencia entre la mujer negra y la oscuridad moral. En las épocas posteriores a la esclavitud, el pelo de la mujer negra hubo de ser "planchado" para atenerse a las prescripciones de la belleza europeizante. En "Pelo”, Morejón celebra su cabello “indomable”, reinscribiéndolo así en la tradición del "afro" y la trenza africana. En su ensayo sobre la poesía de Lucille Clifton, Ajuan Maria Mance habla del sentido lúdico utilizado por Clifton para bregar con la herencia de la esclavitud, enfatizando la capacidad de supervivencia de la mujer negra:

For example, play allows African American Women poets like Clifton to review the tragic history of black women's enslavement and reinterpret it as evidence of endurance, a characteristic that merits pride and celebration. (137)

Revista Iberoamericana, Vol. LXXVII, Núm. 235, Abril-Junio 2011, $461-471$
ISSN 2154-4794 (Electrónico) 
[Por ejemplo, lo lúdico utilizado por las poetas afroamericanas como Clifton les permite revisar la historia trágica de la esclavitud y reinterpretarla como evidencia de su resistencia, característica que merece orgullo y celebración.]

Nancy Morejón expone el cuerpo de la hablante del poema a la luz del lector exigiendo para él un contexto socio-histórico que lo valoriza en su capacidad de convocar una imagen a la vez personal, a la vez trascendental.

Si en "El tambor”, "Pelo” y "Presente Brígida Noyola”, el cuerpo de la mujer negra ha sido presentado en toda su claridad, hay otra poesía asociada con el amor erótico que reside en la penumbra. En su estudio seminal sobre la figura de la mulata en la literatura cubana, Vera Kutzinski comprueba que, particularmente en la poesía cubana del siglo veinte, el cuerpo de la mujer negra se convierte en la representación del deseo masculino y en la subsecuente desaparición de la mujer negra como sujeto capaz del deseo:

The mulata as displayed in the poems I listed at the beginning of this chapter, then, is little more than a body inscribed with and subjected to male desire, sexual and political. That body may be feminine in appearance, but much more significant than its gender attributes is that it is the site of an "erotic" performance represented as feminine. (174)

[La mulata, tal y como ha sido revelada en los poemas que he mencionado al principio de este capítulo, no es nada más que un cuerpo inscrito y sometido al deseo masculino, tanto sexual como político. El cuerpo puede parecer femenino, pero mucho más significante que sus cualidades de género, es el hecho de que constituye el sitio de un espectáculo “erótico" representado como femenino.]

A partir de su poema “Amo a mi amo”, Nancy Morejón deja de representar a la esclava como mujer pasiva incapaz de rebelarse como sujeto histórico: "Amo a mi amo pero todas las noches/ cuando atravieso la vereda florida hacia el cañaveral/donde a hurtadillas hemos hecho el amor,/ me veo cuchillo en mano, desollándole como a una res/ sin culpa" (Looking 198). El cuerpo de la mujer negra, no mulata, no se constituye como "el sitio de un espectáculo" sino como sujeto capaz de blandir el cuchillo libertador. En el momento histórico de la rebelión de la esclava contra su amo, la única mención del cuerpo es para maldecirlo: "Maldigo [...] / estos senos de piedra [...] / este vientre rajado [...] / este maldito corazón” (198). En este momento de rebelión, la mujer negra se ha dado cuenta de que su cuerpo no representa más que el deseo de un amo que la subyuga, y observa su cuerpo como cualidades ajenas al amor: “de piedra”, "rajado”, "maldito”. El cuerpo de la mujer negra deja de poseer atributos "femeninos". Al maldecir su propio cuerpo, la mujer negra lo desviste del erotismo proyectado en él por el amo. Si en la poesía cubana el cuerpo de la mulata, desde Ballagas a Guillén, había de representar un espectáculo erótico (Kutzinski 174), a partir de la poesía de Morejón el cuerpo erótico

Revista Iberoamericana, Vol. LXXVII, Núm. 235, Abril-Junio 2011, $461-471$
ISSN 2154-4794 (Electrónico) 
de la mujer negra ha de habitar los intersticios de la penumbra mediante los vuelos retóricos del símil y la metáfora.

En "A un muchacho" se expresa la sexualidad de la mujer en sólo tres versos: "Entre sus brazos, vivo./ Entre sus brazos duros quise morir/ como un ave mojada". El cuerpo de la mujer existe sólo en su elisión poética a través del símil: "como un ave mojada” (Looking 150). El cuerpo de la mujer está presente, pero sólo en relación al cuerpo visible del hombre:

\author{
Un muchacho del mundo sobre mí \\ Y los cantares de la Biblia \\ modelaron sus piernas, sus tobillos \\ y las uvas del sexo \\ y los himnos pluviales que nacen de su boca (150)
}

En este poema donde el acto sexual queda presentado en toda su claridad(en relación al Cantar de los Cantares), interesa que el cuerpo del hombre quede expuesto ante una tradición erótico-literaria antiquísima, pero el de la mujerno, evitando así la objetivización del cuerpo femenino en el contexto histórico literario. En "A un muchacho", es ella la que observa y desea el cuerpo masculino. También es de notar que el acto sexual ocurre "Entre la espuma y la marea”. Este marco acuático importa, porque Morejón ha de valerse de él para significar el encuentro sexual en otros poemas como "Día del murex” y “Carta náutica” (Cuerda 84, 49) que analizaré en breve.

En su ponencia “Narcisismo poético en Nancy Morejón”, leída en la Universidad de Missouri, Juanamaría Cordones-Cook, citando a Morejón, observa en el poema "Autoformas", "no ha habido un solo espacio para tu nombre” y, por lo tanto, el nombre queda representado por un girasol, "flor que simboliza a Ochún, la orisha del amor, cuya luz la hablante desearía contemplar junto al ser querido frente a otra luz, la de una luna llena”. Aquí vemos cómo el ser amado sólo puede ser visto a la luz de la luna. Así el cuerpo femenino desaparece detrás de la flor que por su propio nombre debería lucirse ante el sol, y no la luna. "Autoformas" no puede nombrar ni el nombre de la amada, ni el nombre del amor que sólo puede ser visto a la luz de la luna:

En este teclado que de pronto vomita prodigios y ansiedad,
no ha habido un solo espacio para tu nombre,
ni para el girasol, marcado por la luz,
que yo quisiera contempláramos junto a otra luz:
la de la luna redonda y clara de esta noche. (citado por Cordones-Cook)

El amor no alumbrado por el sol, el amor que hace girar la flor, queda en la penumbra, entre el deseo del "yo" y aquél de un "nosotros” ("que yo quisiera contempláramos

Revista Iberoamericana, Vol. LXXVII, Núm. 235, Abril-Junio 2011, $461-471$
ISSN 2154-4794 (Electrónico) 
junto a otra luz"). La voz lírica de la hablante necesita del apoyo de la voz de la amante para iluminar el amor que no ha de nombrarse.

En efecto, Nancy Morejón utiliza el lenguaje de las flores como un lenguaje capaz de expresar aquello que reside en la oscuridad. De acuerdo con el estudioso del lenguaje de las flores en su libro Flower Poetics in Nineteenth-Century France, Philip Knight concluye que poetas como Hugo, Baudelaire, Nerval, y luego Mallarmé convierten el lenguaje de las flores en un anti-lenguaje que paradójicamente ofrece y retiene su significado ("an anti-language paradoxically offering and withholding meaning” 246). Esta posibilidad poética de revelar lo que no se debe nombrar fue bien desarrollada por Baudelaire en Les Fleurs du Mal. Knight especifica que la conciencia irónica del poeta francés lo convierte en un poderoso agente de la creación de su propio lenguaje poético de las flores del Mal ("the poet's ironic modern consciousness makes him a powerful agent in the creation of his own poetic language des fleurs du Mal" [244]).

$\mathrm{Al}$ igual que Baudelaire juega con el sentido paradójico del lenguaje de las flores, Morejón acude a los múltiples sentidos del mar para crear su propio lenguaje erótico. En "Día del murex”, las aguas del mar constituyen el "universo más hermoso":

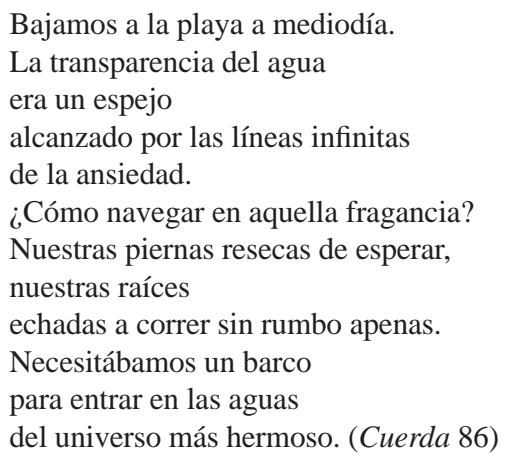

En la playa, a orillas del mar, "las líneas infinitas/ de la ansiedad”, la inquietud sexual, queda reflejada en el espejo del agua. Morejón aquí establecela primera cadena metonímica del poema: la ansiedad se debe al estado reseco de las raíces que son las piernas de los hablantes, quienes reconocen la necesidad de sumergirse en las aguas del deseo. En las estrofas segunda y tercera existen amigos que prefieren quedarse a la orilla del mar: “Oh, las luces de los amigos, varados en la arena” (84), pero el "nosotros" de la hablante pregunta “¿Cómo navegar en aquella fragancia?” Con el segundo juego metonímico del lenguaje del mar, Morejón establece una cadena de relaciones que van de las piernas al barco, al mar, al universo más hermoso. En la próxima estrofa las piernas se convierten en el barco necesario para alcanzar el universo más hermoso del deseo: "Entramos al

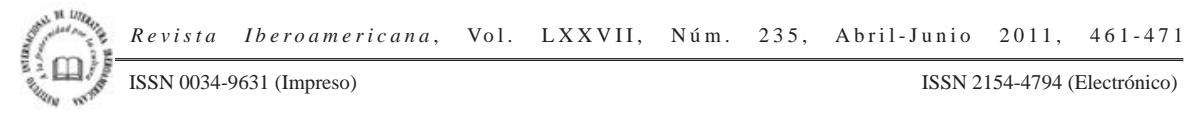


mar. Entramos/ y el canto sin límites de nuestras piernas/ creó el barco en donde nos depositaron" (84). Desde el mar, la voz poética reconoce que ha abandonado la orilla y que la intensificación del deseo ya no permite regreso: "Espumas y espumas en el desierto corazón/ de la ribera. No puedo regresar./ Ya no podemos ir atrás” (85). Nótese el cambio de énfasis del “yo” al "nosotros” para anticipar la sexualidad compartida.

En la última estrofa se hace explícito el significado del nombre del poema. El murex, molusco marino cuyo caparazón sirve de trompeta en las representaciones de los tritones del mar, tiene por extensión una especie de cabello que el poema describe como "encaje insalvable” (85). Con la imagen del murex, Morejón expresa la culminación del deseo:

Entramos a las redes del murex,

al encaje insalvable

de su hendija minúscula,

al punto diabólico de la esperanza

que se incrusta en la piel soñada de las conchas.

Cabalgaban ellos en su ritmo de huracán solitario.

Por fin, alcanzamos el murex, su fija transparencia. (Cuerda 87-88)

Los cuatro primeros versos describen el sexo femenino como "redes del murex" y la culminación del acto sexual como el "punto diabólico de la esperanza". El "ellos" se refiere a aquellos que quedaron en la arena mientras el "nosotros" se ha sumergido en las profundidades del mar para poder alcanzar el murex. El arco de la expresión sexual pasa de la ansiedad a la esperanza mediante el uso del "barco" poético en su "fija transparencia”. Si en el primer poema analizado en este ensayo el cuerpo resuena como un tambor, en "Día del murex", "la piel soñada de las conchas" susurra al son de las olas. "El canto de las piernas" que creó el barco para navegar "en aquella fragancia”, ahora resulta ser el canto o el sonido del murex creado por el aliento del tritón que representa la voz poética de la hablante. En última instancia, "Día del murex" constituye una alegoría del conocimiento sexual ya que las profundidades del mar pueden connotar las diferentes manifestaciones del saber. ${ }^{2}$

"Carta náutica", otro poema erótico en Cuerda veloz proporciona un excelente ejemplo del lenguaje poético que se niega a nombrar el amor sexual al mismo tiempo que hace resaltar los cuerpos de los amantes dentro del contexto marítimo:

2 Para un excelente análisis del mar como símbolo del conocimiento histórico en la poesía de Elizabeth Bishop y Adrienne Rich, ver el artículo de Roger Gilbert, "Framing Water". En mi lectura de "Día del murex”, propongo que Nancy Morejón utiliza el mar como símbolo del saber sexual. Quiero agradecer a mi colega Maria Koundoura en Emerson College el haber sugerido que consultara la obra de Adrienne Rich.

Revista Iberoamericana, Vol. LXXVII, Núm. 235, Abril-Junio 2011, $461-471$
ISSN 0034-9631 (Impreso) 


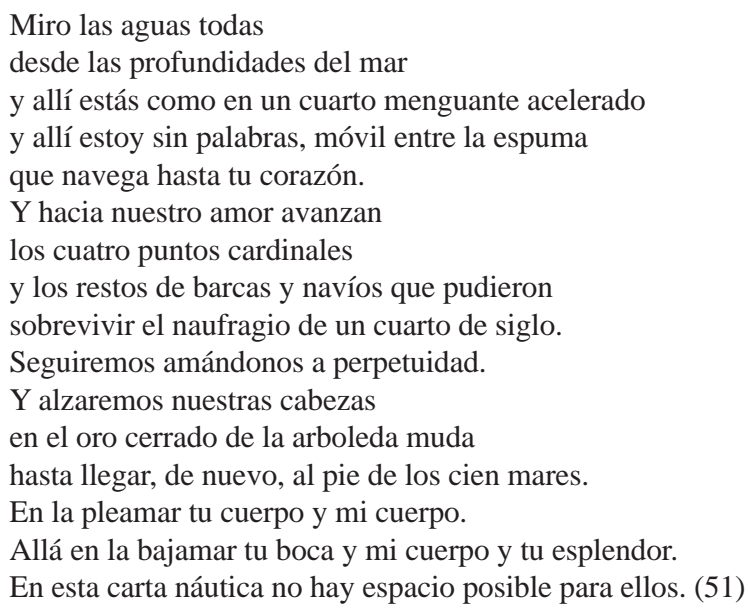

El punto de vista de la voz poética reside "sin palabras" en "las profundidades del mar" (como Yemayá/Olokun, la orisha poderosa de todos los mares, creadora de todo lo que existe). El espacio de la escritura, la carta náutica del último verso, le niega lugar a los cuerpos, pero ellos persisten "al pie de los cien mares". Cuando las palabras se resisten, la espuma "navega hasta tu corazón” para hacer explícito el amor "a perpetuidad". Desde las profundidades del mar, mediante el poder paradójico de su lenguaje marítimo, Nancy Morejón hace resaltar los cuerpos utilizando la sinécdoque: "alzaremos nuestras cabezas". Sólo "al pie de los cien mares" pueden los cuerpos entrelazarse encubiertos entre "la pleamar" y la "bajamar".

Hacia el final de la colección poética Cuerda veloz, Nancy Morejón nos presenta un fragmento de la sección final de "Amor, ciudad atribuída”, aquí titulada "Cuando te amo" (187-88). En su ensayo sobre "Amor, ciudad atribuída”, Diane E. Marting clasifica el poema como ejemplo de la metafísica del erotismo ("a unique and individual metaphysic of eroticism” [201]), donde el objeto del deseo es la ciudad amada. Hablando de la última sección del poema, Marting especifica que el amante, ante la ciudad, ahora puede ser nombrado. Pero, al querer nombrarlo, desaparece en la voz de la hablante: "estás en sustancia y tu nombre se podría/ acariciar con sonrisas negadas hay un número/indecible de tus poros en mí” (187). Al aislar este fragmento final de un poema mayor, Nancy Morejón lo separa del contexto erótico donde el amante se relaciona con la ciudad. Al principio de "Cuando te amo", el cuerpo parece adquirir presencia, "estás en sustancia”, y luego "hay un número/ indecible de tus poros en mí”. Pero el cuerpo, objeto del deseo de la hablante que repite "te amo" tres veces, sólo aparece en la forma singular del verbo, "estás”. También aparece, o se esconde, detrás del pronombre directo "te" en "te amo”. Por fin, en la tercera estrofa, emerge como sujeto: "tú me surges de todo" (187),

Revista Iberoamericana, Vol. LXXVII, Núm. 235, Abril-Junio 2011, $461-471$
ISSN 2154-4794 (Electrónico) 
sugiriendo que el ser querido se manifiesta como una fuente de todo lo que existe. Pero aún así, el objeto del deseo ha sido desplazado detrás de un pronombre, en primer lugar singular, y luego plural: "para los/ que/ no quieran mirar a nuestros lechos les ofrezco esta voz" (188). Al final del poema, los amantes, "nosotros”, han desaparecido en la penumbra de "nuestros lechos" para no ser vistos por los que no quieran mirar. La voz de la hablante insiste en la existencia del amor eróticamente expresado en los lechos con un "te amo" más. Si en los otros poemas eróticos aquí analizados los cuerpos de los amantes aparecen y desaparecen entre las olas del mar y desde las profundidades del mar, en "Cuando te amo" la elipsis de los cuerpos se efectúa gramaticalmente, con el prononmbre "tú" representando al ser querido, y el posesivo "nuestros" desplazando los cuerpos completamente. Si en "A un muchacho" el cuerpo femenino desaparece detrás del símil ("como un ave mojada”), en "Cuando te amo" el cuerpo del ser querido queda elidido por el pronombre "tú".

En su estudio de la poética de Adrienne Rich, Kevin McGuirk afirma que la obra de Rich traza un itinerario basado en las funciones líricas y retóricas de la poesía ("[The] work traverses the line between lyric expression and rhetorical act” [61]). La expresión lírica se entiende aquí por una meditación de la experiencia subjetiva dirigida a un recipiente "tú" o "usted” no nombrado (McGuirk 82), mientras que la expresión retórica insiste en un lenguaje dirigido hacia un público en su contexto social, histórico, político (McGuirk 62). A manera de conclusión quisiera yo también proponer que la obra de Morejón, particularmente aquella que concierne a la representación del cuerpo de la mujer negra, ensaya el registro retórico cuando su cuerpo/tambor hace resonar los contextos históricos de la mujer negra (“El tambor”, "Pelo”y “Presente Brígida Noyola”). La poesía erótica de Morejón transforma el yo histórico/mítico en un "nosotros” íntimo que habla mediante el susurro de las olas del conocimiento sexual ("Día del murex" y "Carta naútica"). En ambos casos, el cuerpo de la mujer negra es la hablante del poema, así evitando que su cuerpo se convierta "en un espectáculo con otros fines” (Bennett y Dickerson 5); estos fines aludirían a la explotación sexual del cuerpo de la mujer negra desde la esclavitud y desde una tradición literaria que lo convierte en un espectáculo erótico (Kutzinski 174).

Es notable observar que en "A un muchacho" la voz poética habla desde el "yo" que observa a su amante ("Entre sus brazos vivo” [150]), replicando así la función lírica tradicional que expresa una subjetividad singular. Sin embargo, en sus poemas eróticos donde se deja de nombrar el objeto del deseo más allá del pronombre "tú", el "yo" se convierte en "nosotros” para ubicar los cuerpos en los márgenes de la visibilidad (“Entramos a las redes del murex” [85]). El verso erótico de Nancy Morejón se sitúa entonces entre el registro íntimo de la poesía lírica dirigida a un amante o específico o universal, y el registro retórico que habla a una comunidad más abarcadora, en este caso, a todo lector que entienda el significado del lenguaje de las flores y del mar.

\footnotetext{
Revista Iberoamericana, Vol. LXXVII, Núm. 235, Abril-Junio 2011, $461-471$
ISSN 0034-9631 (Impreso)
} 
BiBLIOGRAFÍA

Bennett, Michael y Vanessa D. Dickerson, eds. Introducción. Recovering the Black Female Body: Self-Representations by African American Women. New Brunswick: Rutgers UP, 2000. 1-15.

Cabrera, Lydia. El monte (Igbo, Finda, Ewe Orisha, Vitti Nfinda). Miami: Colección del Chicherekú, 1992.

Cordones-Cook, Juanamaría. "Narcicismo poético en Nancy Morejón”. Conference on Afro-Romance Film and Culture. Afro-Romance Institute. University of MissouriColumbia. 3-4 abril 2008.

Gilbert, Roger. "Framing Water: Historical Knowledge in Elizabeth Bishop and Adrienne Rich.” Twentieth Century Literature 43/2 (verano 1997): 144-61.

Knight, Philip. Flower Poetics in Nineteenth-Century France. Oxford: Clarendon, 1986.

Kutzinski, Vera M. Sugar's Secrets: Race and the Erotics of Cuban Nationalism. Charlottesville: U of Virginia P, 1993.

Mance, Ajuan María. "Re-locating the Black Female Subject: The Landscape of the Body in the Poems of Lucille Clifton." Recoverning the Black Female Body: Self-Repesentations by African American Women. Michael Bennett y Vanessa D. Dickerson, eds. New Brunswick: Rutgers UP, 2000. 123-40.

Marting, Diane E. “The Representation of Female Sexuality in Nancy Morejón's Amor, ciudad atribuída, poemas”. Singular Like a Bird: The Art of Nancy Morejón. Miriam deCosta-Willis, ed. Baltimore: Howard UP, 1999. 201-208.

Matibag, Eugenio. Afro-Cuban Religious Experience: Cultural Reflections in Narrative. Gainesville: UP of Florida, 2001.

McGuirk, Kevin. "Philoctetes Radicalized: 'Twenty-One Love Poems' and the Lyric Career of Adrienne Rich.” Contemporary Literature 34/1 (primavera 1993): 61-87.

Morejón, Nancy. Carbones silvestres. La Habana: Letras Cubanas, 2005. Cuerda veloz: antología poética, 1962-1992. La Habana: Letras Cubanas, 2002. Looking Within/Mirar adentro. Juanamaría Cordones-Cook, ed. Detroit: Wayne State UP, 2003.

Rooks, Noliwe. "Wearing Your Race Wrong: Hair, Drama, and a Politics of Representation for African American Women at Play on a Battlefield.” Fotografías por Bill Gaskins. Recovering the Black Female Body: Self Representations by African American Women. Michael Bennett y Vanessa D. Dickerson, eds. New Brunswick: Rutgers UP, 2000. 279-95.

\footnotetext{
ISSN 0034-9631 (Impreso)
} 
